\title{
Evaluación de la función renal del donante: TFG, proteinuria, hematuria y urolitiasis. ¿Cómo evaluar? ¿Quién puede donar?
}

\author{
Donor renal function evaluation: GFR, \\ proteinuria, hematuria and urolithiasis. \\ How to evaluate it? Who can become a donor? \\ Roxana Villca-Gonzáles* \\ * Servicio de Nefrología. Hospital Regional «Lic. Adolfo López Mateos», ISSSTE.
}

\section{EVALUACIÓN}

- La evaluación de la función renal del donador se realizará con la determinación de la tasa de filtrado glomerular (TFG).

- De forma inicial, la TFG se determinará con el cálculo de la TFG estimada por creatinina (eTFGcr) por medio de: CKD-EPI creatinina.

- Debe realizarse una nueva determinación confirmatoria de la TFG utilizando el método más exacto del que disponga el centro, puede ser alguno de los siguientes:

- Repetir el cálculo de la TFG estimada por creatinina (eTFGcr) por medio de: CKD-EPI creatinina o CKD-EPI cistatina-creatinina.

- Medición de la TFG (mTFG) por medio de inulina, iotalamato, 51Cr-EDTA, iohexol o 99mTcDTPA.

- El gammagrama renal sólo se recomienda en casos en los que exista anormalidades parenquimatosas, vasculares, urológicas o asimetría $>10 \%$ en el tamaño renal por estudios de imagen, con el fin de determinar la función renal individualizada por riñón. Deben utilizarse agentes que se excretan por filtración glomerular (99mTc-DTPA).
NIVEL DE TFG

- Para la donación se recomienda tener una TFG $\geq$ $90 \mathrm{~mL} / \mathrm{min} / 1.73 \mathrm{~m}^{2}$.

- Es contraindicación absoluta de donación una TFG $<60 \mathrm{~mL} / \mathrm{min} / 1.73 \mathrm{~m}^{2}$.

- En caso de tener una TFG entre $60-90 \mathrm{~mL} /$ $\mathrm{min} / 1.73 \mathrm{~m}^{2}$, el caso debe ser individualizado, considerando edad, comorbilidades y riesgos potenciales.

- Cuando exista asimetría en la TFG, anormalidades parenquimatosas, vasculares o urológicas se debe considerar utilizar en la donación el riñón más afectado.

\section{SEGUIMIENTO}

- Posterior a la donación, el riesgo absoluto de enfermedad renal crónica a lo largo de la vida es muy pequeño tomando en cuenta a la población general. Esta situación debe ser consentida por el donador.

- Posterior a la cirugía se recomienda que el donador tenga seguimiento médico a lo largo de toda su vida para realizar la evaluación de su función renal. 
El nivel de la tasa de filtración glomerular (TFG) es ampliamente aceptada como el mejor índice general de la función renal en salud y en enfermedad. En la población general la disminución de la TFG está asociada con un mayor riesgo de falla renal crónica, enfermedad cardiovascular y muerte..$^{1,2}$

La TFG normal es aproximadamente de $130 \mathrm{~mL} /$ $\mathrm{min} / 1.73 \mathrm{~m}^{2}$ en hombres y $120 \mathrm{~mL} / \mathrm{min} / 1.73 \mathrm{~m}^{2}$ en mujeres hasta los 25 años, con considerable variación entre individuos de acuerdo con la edad, sexo, tamaño corporal, entre otros. Para estandarizar la TFG se debe realizar el ajuste al área de superficie corporal. Posterior a los 40 años la tasa promedio de disminución de la TFG es aproximadamente $0.75 \mathrm{~mL} / \mathrm{min} / \mathrm{año}$, existe una variabilidad amplia y las fuentes de esta variación todavía no están del todo establecidas. ${ }^{1}$

Las guías KDIGO 2012 CKD recomiendan que la función renal se exprese como TFG en $\mathrm{mL} / \mathrm{min} / 1.73 \mathrm{~m}^{2}$ y que en la evaluación se realice la determinación inicial de la TFG, así como una determinación confirmatoria. ${ }^{3}$

La TFG no puede ser medida directamente en humanos, por lo que la TFG real no se conocerá con certeza. Lo que sí se puede obtener actualmente es una TFG medida indirectamente (mTFG) (que se evalúa de manera indirecta a través de la depuración de marcadores de filtración exógenos); y una TFG estimada (eTFG) (que se evalúa a través de niveles séricos de solutos endógenos y ecuaciones de estimación). ${ }^{4}$

El estándar de oro para determinar la TFG medida (mTFG) es utilizar la depuración urinaria de un marcador de filtración ideal (sustancia que es libremente filtrado en el glomérulo) como es la inulina. Se han propuesto muchos marcadores de filtración y métodos de depuración alternativos, pero todos son difíciles de llevar a cabo o tienen un costo alto, por lo que no se puede acceder a ellos. Entre los marcadores de medicina nuclear están 51Cr-EDTA y 99mTc-DTPA, éstos son considerados ya que tienen una adecuada precisión. La depuración de creatinina con orina de 24 horas, tiene muchas limitaciones para la evaluación de la TFG por los errores de precisión probablemente relacionada a fallas en la recolección de orina, así mismo, aunque este correcta la recolección, la depuración de creatinina puede sobreestimar la TFG en un $10-15 \%$ por la secreción tubular de creatinina. 5,6

Para el cálculo de la TFG estimada (eTFG) se utilizan fórmulas que usan el nivel sérico de creatinina y/o cistatina $\mathrm{C}$. El nivel de creatinina puede modificarse por la masa muscular del paciente y/o por su dieta; asimismo, es fundamental remarcar que en el laboratorio el ensayo para la determinación de creatinina debe estar de acuerdo con el estándar de referencia internacional. ${ }^{7}$

Se recomienda utilizar la ecuación CKD-EPI para la eTFG basada en creatinina, esta ecuación fue desarrollada en personas con y sin enfermedad renal, diabetes, donadores y receptores renales, se tomó en cuenta raza blanca, negra, hispanos y asiáticos. Incluye edad, raza, género y creatinina sérica; los resultados son confiables en la mayor parte del rango de TFG. Es importante señalar que su sesgo es menor en TFG mayores a 60 $\mathrm{mL} / \mathrm{min} / 1.73 \mathrm{~m}^{2}$ en comparación a otras ecuaciones. ${ }^{8}$ La fórmula de Cockcroft-Gault y MDRD no son precisas en TFG mayores a $60 \mathrm{~mL} / \mathrm{min} / 1.73 \mathrm{~m}^{2}$, por lo que no se recomendaría su uso en potenciales donadores. ${ }^{9}$

La cistatina $C$ es una proteína producida por las células nucleadas y es distribuida en el líquido extracelular. Cerca de $99 \%$ de la cistatina C es reabsorbida por las células tubulares proximales. Los valores de cistatina $\mathrm{C}$ son afectados por el tabaquismo, inflamación, adiposidad, enfermedades tiroideas, neoplasias malignas y uso de glucocorticoides. ${ }^{10}$ Aunque ya existe material de referencia para la estandarización de su medición, existe aún variaciones considerables entre los ensayos, lo cual limita su uso. ${ }^{11}$

La ecuación para la eTFG basada en cistatina no es más exacta que la ecuación de eTFG basada en creatinina, pero la ecuación de eTFG basada en ambos marcadores es más precisa que las ecuaciones que los utilizan por separado..$^{12,13}$

Por todo lo anterior, en la evaluación inicial del donador, se recomienda determinar la eTFG basada en creatinina sérica (eTFGcr) utilizando la ecuación CKD-EPI. ${ }^{14}$

Debe realizarse una nueva medición confirmatoria de la TFG, utilizando el método más exacto que disponga el centro, pudiendo ser alguno de los siguientes:

- Repetir el cálculo de la TFG estimada por creatinina (eTFGcr) por medio de CKD-EPI creatinina o CKD-EPI cistatina-creatinina.

- Medición de la TFG (mTFG) por medio de inulina, iotalamato, 51Cr-EDTA, iohexol o 99mTc-DTPA.

Entre las ventajas de utilizar una ecuación de estimación de la TFG basada en creatinina están el bajo costo, la amplia disponibilidad y el ensayo estandarizado. ${ }^{4}$

Existe una amplia variación entre las guías actuales en sus recomendaciones para el uso de medicina nuclear como prueba confirmatoria de TFG. Algunas consideran su uso obligado en la evaluación y la mayoría cuando los valores de TFG son limítrofes o no 
concluyentes. ${ }^{14-17}$ En contraste la OPTN requiere que la confirmación de la TFG sea con un radioisótopo o con depuración de creatinina. ${ }^{18}$

Para la donación se recomienda tener una TFG > $90 \mathrm{~mL} / \mathrm{min} / 1.73 \mathrm{~m}^{2}$. Es contraindicación absoluta de donación una TFG $<60 \mathrm{~mL} / \mathrm{min} / 1.73 \mathrm{~m}^{2}$. En caso de tener una TFG entre $60-90 \mathrm{~mL} / \mathrm{min} / 1.73 \mathrm{~m}^{2}$, el caso debe ser individualizado, considerando edad, comorbilidades y riesgos potenciales. ${ }^{14}$

Recientemente se diseñó una calculadora en línea que determina la probabilidad postest de un individuo para un umbral determinado de mTFG basado en eTFG y otros datos demográficos. ${ }^{19,20}$

El gammagrama renal sólo se recomienda en casos en los que exista anormalidades parenquimatosas, vasculares, urológicas o asimetría $>10 \%$ en el tamaño renal por estudios de imagen, esto para determinar la función renal individualizada por riñón. Deben utilizarse agentes que se excretan por filtración glomerular (99mTc-DTPA) cuando exista asimetría en la TFG, anormalidades parenquimatosas, vasculares 0 urológicas y se debe considerar utilizar en la donación el riñón más afectado. ${ }^{15}$

\section{RIESGO DE ERC POSTERIOR A LA DONACIÓN}

Posterior a la nefrectomía el donador sufre una reducción absoluta de $25-40 \%$ en la TFG comparado con su nivel previo a la donación. ${ }^{21,22}$ Si bien está descrito que existe un incremento gradual en la TFG de los donadores, ${ }^{23}$ a los tres años postdonación la TFG de los donadores será menor a los controles sanos no donadores.

Existen varios reportes que muestran que comparados con la población general, los donadores no tienen un riesgo mayor de ERCT; ;3-25 sin embargo, comparados con población sana, los donadores presentan un riesgo relativo de ERCT discretamente mayor. ${ }^{26,27}$ Finalmente, el riesgo absoluto de ERCT continúa siendo bajo, con una incidencia a 15 años de $0.2-0.5 \%{ }^{26-28}$ Este riesgo debe ser explicado y consentido por el donador. Por lo anterior, se recomienda que el donador tenga seguimiento médico a lo largo de toda su vida para realizar la evaluación de su función renal.

\section{PROTEINURIA EN EL DONANTE}

\section{Evaluación}

- Debe realizarse una evaluación inicial y una prueba confirmatoria.
- La valoración inicial puede realizarse con el índice albúmina/creatinina en una muestra aislada de orina o con albuminuria en orina de 24 horas. Debido a la falta de estandarización para la medición de proteinuria se recomienda de preferencia realizar la medición de albuminuria.

- La prueba confirmatoria puede realizarse con la medición de albuminuria en orina de 24 horas (mg/ día). También se puede realizar la prueba confirmatoria con una nueva determinación del índice albúmina/creatinina en muestra aislada de orina.

\section{NIVEL DE PROTEINURIA}

- Para la donación se recomienda una albuminuria $<30 \mathrm{mg} /$ día o índice albuminuria/creatinuria < 30 $\mathrm{mg} / \mathrm{g}$; proteinuria $<150 \mathrm{mg} / 24$ horas o índice proteinuria/creatinuria $<150 \mathrm{mg} / \mathrm{g}$.

- Es contraindicación para la donación: albuminuria $>100 \mathrm{mg} / 24$ horas y/o proteinuria > $300 \mathrm{mg} / 24$ horas.

- En caso de albuminuria entre $30-100 \mathrm{mg} / 24$ horas y/o proteinuria $150-300 \mathrm{mg} / 24$ horas se debe realizar un abordaje individualizado.

- Ante la presencia de proteinuria, primero deben descartarse causas de proteinuria transitoria: fiebre, infección de vías urinarias, ejercicio intenso y proteinuria ortostática.

Es bien conocido que la albuminuria y proteinuria son marcadores de daño renal y son un criterio fundamental en la definición de enfermedad renal crónica; asimismo, están asociados con enfermedad cardiovascular y muerte. ${ }^{2,3}$

La albuminuria mide la concentración de albúmina en orina que refleja un incremento en la permeabilidad en el glomérulo (de origen glomerular). La proteinuria mide proteínas de alto peso molecular (incluida la albúmina), proteínas de bajo peso molecular (que denota baja reabsorción a nivel del túbulo, proteinuria tubular) y proteínas secretadas por el tracto urinario. La determinación de albuminuria es el método preferido para la evaluación de la función renal, en virtud de que la medición de proteinuria no puede ser estandarizada. ${ }^{29}$

Las guías de KDIGO de enfermedad renal crónica recomiendan que se realice la medición inicial y la medición confirmatoria de albuminuria. ${ }^{3}$ Siempre se prefiere la medición en la mañana. Además, recomiendan que se utilice el índice albuminuria/creatinuria de forma inicial, puesto que si bien el estándar de oro es 
la medición de albuminuria en orina de 24 horas, la recolección de orina es altamente inexacta llevando a un error en la determinación de albuminuria. ${ }^{30}$ La medición del índice albúmina/creatinina en muestra aislada de orina matutina es un buen predictor y se correlaciona con la medición de albuminuria en orina de 24 horas. ${ }^{31}$

Para la prueba confirmatoria se recomienda realizar la medición de la tasa de excreción de albúmina (mg/24 horas) y si no se logra ésta se puede repetir el índice albuminuria/creatinuria en muestra aislada. ${ }^{14}$

Son causas de un incremento en albuminuria de forma transitoria: contaminación con menstruación, infección de vías urinarias, ejercicio, proteinuria ortostática. ${ }^{29}$

Un índice albuminuria/creatinuria $<30 \mathrm{mg} / \mathrm{g}$ es normal a levemente incrementado; entre $30-300 \mathrm{mg} / \mathrm{g}$ es moderadamente incrementado y $>300 \mathrm{mg} / \mathrm{g}$ es severamente incrementado. Un nivel de albuminuria entre 5-10 $\mathrm{mg} / 24$ horas es normal en jóvenes sanos, este nivel se incrementará con la edad. Un nivel $<30 \mathrm{mg} /$ día es considerado como normal a levemente incrementado. Un nivel de albuminuria > $30 \mathrm{mg} /$ día es moderadamente incrementado y refleja una alteración en la estructura de la pared del capilar glomerular (microalbuminuria), y un nivel mayor a 300 mg/día es considerado severamente incrementado (macroalbuminuria). ${ }^{3}$ Recordar que la albuminuria $>30 \mathrm{mg} / 24$ horas por tres meses o más satisface los criterios de enfermedad renal crónica.

El nivel de proteinuria $<150 \mathrm{mg} / 24$ horas se considera como nivel normal a levemente incrementado; moderadamente incrementado entre $150-500 \mathrm{mg} / 24$ horas y severamente incrementado a valores $>500$ $\mathrm{mg} / 24$ horas. $^{3}$

Recientemente las Guías KDIGO 2017 recomiendan que se mida albuminuria en vez de proteinuria. Utilizando el índice albuminuria/creatinuria en muestra aislada como prueba inicial, seguido de una prueba confirmatoria de albuminuria/24 horas, ya sea con recolección de orina de 24 horas o una segunda determinación de índice albuminuria/creatinuria si la recolección no se puede realizar. ${ }^{14}$ Además, estas guías sugieren que un nivel de albuminuria $<30 \mathrm{mg} / 24$ horas es normal para la donación. Un valor de albuminuria > 100 mg/día es contraindicación para la donación. Un valor entre 30-100 mg/día debe individualizarse basado en la edad y valores aceptados en la Unidad de Trasplante. Si bien este valor se podría considerar como enfermedad renal crónica, el riesgo de ERCT estimado predonación es muy bajo en ausencia de disminución de TFG y otros factores de riesgo. ${ }^{32}$
Previamente, el Foro de Ámsterdam consideraba como exclusión a la donación una proteinuria > $300 \mathrm{mg} / 24$ horas. Al realizar una evaluación de los motivos de rechazo a donadores en centros de Estados Unidos se encontró que los centros utilizaban como punto de corte una proteinuria $>150 \mathrm{mg} / 24$ horas. ${ }^{16,33}$

Las guías británicas 2018 consideran como contraindicación absoluta un índice albuminuria/creatininuria $>300 \mathrm{mg} / \mathrm{g}$, índice proteinuria/creatinuria $>500 \mathrm{mg} / \mathrm{g}$, albuminuria $>300 \mathrm{mg} / 24$ horas y proteinuria $>500$ $\mathrm{mg} / 24$ horas. Asimismo, colocan como contraindicación relativa un índice albuminuria $30-300 \mathrm{mg} / 24$ horas y proteinuria $150-500 \mathrm{mg} / 24$ horas ${ }^{15}$

\section{HEMATURIA}

\section{Evaluación}

- Debe evaluarse la presencia de hematuria en todos los posibles donadores.

- Ante la presencia de hematuria microscópica persistente debe descartarse: causas benignas, infección de vías urinarias, litiasis renal, anormalidades en el tracto urinario y procesos neoplásicos.

- Si todos estos estudios son negativos o si se encuentra eritrocitos dismórficos se debe valorar realizar biopsia renal percutánea para buscar patología glomerular.

- La donación está contraindicada en caso de anormalidades glomerulares en la biopsia o en caso de enfermedad maligna en el tracto urinario.

- Si se detecta etiología benigna se puede realizar el trasplante; sin embargo, será importante dar seguimiento estrecho tanto del receptor como en el donador.

La prevalencia de hematuria microscópica va de $3-20 \%$, esta variabilidad depende de la edad, género y otras características de los pacientes. La mayor frecuencia es evidente en hombres adultos mayores con antecedente de tabaquismo. ${ }^{34}$ Hematuria microscópica se define como la presencia de $>3-5$ eritrocitos/ campo en 2-3 ocasiones separadas. ${ }^{35,36}$

Las causas no glomerulares de hematuria microscópica pueden ser: causas benignas (menstruación, ejercicio, hipertrofia prostática benigna), infección de vías urinarias, litiasis y anormalidades en el tracto urinario (incluidas neoplasias). ${ }^{14,34}$ Las causas glomerulares de hematuria microscópica en orden de frecuencia son: nefropatía por IgA (23-45\%), enfermedad 
de membranas delgadas, enfermedades hereditarias como síndrome de Alport. ${ }^{37}$

Las recomendaciones para la evaluación de la hematuria asintomática radican en: evaluación de los factores de riesgo para neoplasias del tracto urinario, evaluación radiológica y cistoscopia en mayores de 35 años. ${ }^{34}$ La incidencia de patología maligna en menores de 40 años es $<1.5 \%$. 38,39

La presencia de eritrocitos dismórficos en la orina sugiere etiología glomerular y si el resto del abordaje resulta negativo sugiere la realización de una biopsia renal. ${ }^{34}$

La donación está contraindicada en caso de anormalidades glomerulares en la biopsia o en caso de enfermedad maligna en el tracto urinario. La nefropatía por IgA es una enfermedad que puede progresar a ERC, ${ }^{40}$ así como el síndrome de Alport; por otro lado, aunque algunas guías permiten la donación en pacientes con enfermedad de membranas delgadas existen reportes que sugieren riesgo de hipertensión y proteinuria en este grupo de pacientes comparados con la población general, ${ }^{41}$ por lo que su aceptación como potenciales donadores debe ser tomado con precaución.

\section{UROLITIASIS DEL DONANTE}

- En todo candidato a donador renal debe investigarse la presencia y antecedentes de litiasis renal, ya que la principal preocupación es la recurrencia en el riñón remanente.

- En todo potencial donador con antecedente o episodio actual de litiasis debe buscarse la causa subyacente.

- Debe contraindicarse la donación si existe alto riesgo de recurrencia de litiasis o tienen alguna enfermedad metabólica.

- Podrá aceptarse como donador si tiene bajo riesgo de recurrencia; tuvo un episodio único de litiasis mayor a 10 años, o el lito actual es único y pequeño, no tiene anormalidades metabólicas u otra condición asociada con la formación de litos recurrentes.

En todo candidato a donador renal debe investigarse la presencia de litiasis renal (sintomática o asintomática) y los antecedentes de litiasis, puesto que no es un hallazgo infrecuente y las implicaciones a futuro en el donador son importantes. En la actualidad muchos centros de trasplantes a nivel mundial han flexibilizado sus criterios para aceptar a potenciales donadores con litiasis, pero en nuestro entorno donde el seguimiento del donador no es regular esta flexibilidad debe tomarse con precaución.

La prevalencia global de litiasis sintomática ha tenido un incremento paulatino en las últimas décadas, hoy en día se estima en $8.8 \%$ en la población general, siendo mayor en los hombres (10.6\%) que en las mujeres $(7.1 \%)$. La prevalencia varía a lo largo de la vida; alrededor de los 20 años es de $3.1 \%$ (en hombres y mujeres) y alcanza a los 60 años a $19.1 \%$ en hombres y $9.4 \%$ en mujeres. ${ }^{42}$

La litiasis asintomática no es infrecuente, en un estudio que realizó tomografía no contrastada a una población asintomática encontró una prevalencia de litiasis de $7.8 \%$ (hombres $9.7 \%$ y mujeres $6.3 \%$ ) con un promedio de 2.1 litos por caso y con un tamaño promedio de $3.0 \mathrm{~mm}$ de diámetro. Al menos $20 \%$ de estos casos experimentaron un episodio sintomático en un periodo de 10 años. La presencia de diabetes y obesidad se asoció al desarrollo de síntomas a lo largo del tiempo. ${ }^{43}$

En una cohorte de potenciales donadores renales ( $n=1,957)$ que se sometieron a una tomografía por el protocolo de trasplante renal encontraron que 3\% tenían antecedente de litiasis asintomática y que $11 \%$ tenía litos presentes en la tomografía (10\% asintomáticos y $1 \%$ sintomáticos). Los donadores asintomáticos tenían un diámetro de los litos menor a los sintomáticos (1.6 mm vs $4.8 \mathrm{~mm}$ ) y no presentaron características usuales en pacientes con litiasis sintomática (como ser de edad mayor, género masculino, hipertensión, obesidad, síndrome metabólico, hiperuricemia, hipercalcemia o hipofosfatemia), lo que sugeriría un mecanismo patofisiológico diferente. ${ }^{44}$ Otras cohortes de potenciales donadores han reportado prevalencia de litiasis asintomática de $5 \%$ (diámetro $2-12 \mathrm{~mm}$ ), ${ }^{45} 5.6 \%{ }^{46}$ y $7.4 \%$ (diámetro $1-9$ $\mathrm{mm}) .{ }^{47}$

Aproximadamente $80 \%$ de los litos renales están compuestos por oxalato de calcio unido a fosfato de calcio, $9 \%$ por ácido úrico, $10 \%$ por estruvita y $1 \%$ por otras causas (cistina, medicamentos). ${ }^{48}$

Si bien se ha demostrado que la litiasis renal como causa primaria de ERCT es infrecuente (alrededor de $3 \%$ de todos los casos).$^{49}$ El riesgo de desarrollar enfermedad renal crónica es mayor en pacientes con litos sintomáticos en comparación a población sin tendencia a formar litos. ${ }^{50,51} \mathrm{La}$ litiasis debida a malformaciones urológicas, trastornos genéticos y síndromes de malabsorción tiene el mayor riesgo de desarrollar enfermedad renal crónica y enfermedad renal crónica terminal. ${ }^{52}$ 
En los donadores que tengan antecedentes de litiasis o con evidencia actual de litiasis se sugiere realizar la siguiente evaluación: ${ }^{53}$

- Evaluación clínica completa: historial médico, historia dietética. Evaluar estudios de imagen disponibles para ver características de los litos.

- Medición de hormona paratiroidea (principalmente si se sospecha de hiperparatiroidismo primario).

- En caso de contar con el lito, realizar análisis del mismo.

- Estudio metabólico:

- En sangre: creatinina, calcio, fósforo, sodio, potasio, bicarbonato, ácido úrico, hormona paratiroidea.

- En orina de 24 horas: calcio, oxalatos, ácido úrico, citratos, sodio, fósforo, potasio y creatinina.

En la actualidad, ante la escasa evidencia que guie la toma de decisiones, existe falta de unanimidad entre las recomendaciones actuales con respecto a la permisibilidad de donación en pacientes con litiasis.

El Foro de Ámsterdam permite la donación de personas con antecedente de episodio único de litiasis sintomática antiguo, así como de donadores con litiasis actual asintomática menor a $1.5 \mathrm{~cm}$ de tamaño, sin anormalidades metabólicas. ${ }^{16}$ Las Guías de la Sociedad Británica aceptan como donadores a pacientes sin patología metabólica significativa concomitante con historia de litiasis o litos pequeños. ${ }^{15}$ Las Guías KDIGO indican que la aceptación de un donador con un lito actual o previo dependerá de la evaluación del riesgo de recurrencia y del conocimiento de las posibles consecuencias de un episodio de litiasis posterior a la donación. ${ }^{14}$

En general, los donadores con antecedente o episodio actual de litiasis son excluidos de la donación si son formadores recurrentes o tienen alguna enfermedad metabólica durante la evaluación:

- Enfermedades congénitas: cistinuria, hiperoxaluria primaria.

- Hipercalciuria, hiperuricosuria, hiperfosfaturia, hipocitraturia, acidosis tubular renal.

- Litiasis secundaria a enfermedad inflamatoria intestinal, sarcoidosis.

- Litiasis por estruvita.

- Nefrocalcinosis, litiasis bilateral o antecedente de recurrencia a pesar de medidas de prevención.
Se consideran características asociadas a mayor riesgo de recurrencia a lo largo de la vida: edad menor a 40 años en el primer episodio, historia familiar de litiasis, recurrencia frecuente de litos. Se consideran características asociadas con menor riesgo de recurrencia a lo largo de la vida: edad mayor a 40 años en el primer episodio, sin sintomatología previa de litiasis renal, lito menor de $15 \mathrm{~mm}$ de diámetro, solitario y unilateral. ${ }^{14}$

Puede aceptarse como donador si tiene antecedente de litiasis y existe bajo riesgo de recurrencia: con un episodio único de litiasis mayor a 10 años y ausencia de anormalidades metabólicas asociadas con la formación de litos (hipercalciuria, hiperuricosuria, hiperoxaluria, hipocitraturia, acidosis metabólica).

Existe cada vez más reportes de centros que utilizan donadores con litos asintomáticos pequeños y unilaterales, sin evidencia de anormalidades metabólicas; según estos reportes tienen una baja progresión a litiasis sintomática (menor de 1\%) aunque los tiempos de seguimiento reportados son cortos..$^{45,47,54} \mathrm{Si}$ la donación procede se recomienda realizar la nefrectomía del riñón que contiene el lito.

Si el lito es muy pequeño $(<4 \mathrm{~mm})$ algunos autores recomiendan dejarlo en su sitio y mantener en vigilancia estrecha. Estos litos tienen tasas altas de paso espontáneo posterior al trasplante, independiente de su localización. ${ }^{55} \mathrm{Si}$ el lito es de mayor tamaño o potencialmente extraíble existen nuevas técnicas quirúrgicas endourológicas mínimamente invasivas que permiten inspeccionar el sistema colector y remover cualquier lito ex vivo antes de implantar el riñón, con una baja morbilidad y bajas tasas de recurrencia. ${ }^{56}$

\section{REFERENCIAS}

\section{EVALUACIÓN DE LA FUNCIÓN RENAL DEL DONANTE}

1. Inker LA, Levey AS. Assessment of glomerular filtration rate. In: Feehally J, Floege J, Tonelli M, Johnson RJ. Comprehensive clinical nephrology. 6th ed. Elsevier; 2019. pp. 29-38.

2. Matsushita K, van der Velde M, Astor BC, Woodward M, Levey AS, de Jong PE et al. Association of estimated glomerular filtration rate and albuminuria with all-cause and cardiovascular mortality in general population cohorts: a collaborative metaanalysis. Lancet. 2010; 375 (9731): 2073-2081.

3. Kidney Disease: Improving Global Outcomes (KDIGO) CKD Work Group. KDIGO 2012 clinical practice guideline for the evaluation and management of chronic kidney disease. Kidney Int. 2013; 1-150.

4. Inker LA, Huang N, Levey AS. Strategies for assessing GFR and albuminuria in the living kidney donor evaluation. Curr Transplant Rep. 2017; 4 (1): 13-23.

5. Stevens LA, Levey AS. Measured GFR as a confirmatory test for estimated GFR. J Am Soc Nephrol. 2009; 20 (11): 2305-2313. 
6. Soveri I, Berg UB, Bjork J, Elinder CG, Grubb A, Mejare I et al. Measuring GFR: a systematic review. Am J Kidney Dis. 2014; 64 (3): 411-424.

7. Myers GL, Miller WG, Coresh $\mathrm{J}$ et al. Recommendations for improving serum creatinine measurement: a report from the Laboratory Working Group of the National Kidney Disease Education Program. Clin Chem. 2006; 52 (1): 5-18.

8. Levey AS, Stevens LA, Schmid CH, Zhang YL, Castro 3rd AF, Feldman $\mathrm{HI}$ et al. A new equation to estimate glomerular filtration rate. Ann Intern Med. 2009; 150 (9): 604-612.

9. Earley A, Miskulin D, Lamb EJ et al. Estimating equations for glomerular filtration rate in the era of creatinine standardization: a systematic review. Ann Intern Med. 2012; 156 (11): 785-795.

10. Keddis MT, Amer H, Voskoboev $\mathrm{N}$ et al. Creatinine-based and cystatin C-based GFR estimating equations and their non-GFR determinants in kidney transplant recipients. Clin J Am Soc. 2016; 11 (9): 1640-1649.

11. Eckfeldt JH, Karger AB, Miller WG, Rynders GP, Inker LA. Performance in measurement of serum cystatin $C$ by laboratories participating in the College of American Pathologists 2014 CYS survey. Arch Pathol Lab Med. 2015; 139 (7): 888-893.

12. Inker LA, Schmid $\mathrm{CH}$, Tighiouart H, Eckfeldt JH, Feldman HI, Greene $\mathrm{T}$ et al. Estimating glomerular filtration rate from serum creatinine and cystatin C. N Engl J Med. 2012; 367 (1): 20-29.

13. Fan L, Inker LA, Rossert J, Froissart M, Rossing P, Mauer M et al. Glomerular filtration rate estimation using cystatin $\mathrm{C}$ alone or combined with creatinine as a confirmatory test. Nephrol Dial Transplant. 2014; 29 (6): 1195-1203.

14. Lentine KL, Kasiske BL, Levey AS, Adams PL, Alberú J, Bakr MA et al. KDIGO Clinical Practice Guideline on the Evaluation and Care of Living Kidney Donors. Transplantation. 2017; 101 (8S Suppl 1): S1-S109.

15. Andrews PA, Burnapp L. British Transplantation Society / Renal Association UK Guidelines for Living Donor Kidney Transplantation 2018: Summary of Updated Guidance. Transplantation. 2018; 102 (7): e307.

16. Delmonico F, Council of the Transplantation Society. A report of the Amsterdam forum on the care of the live kidney donor: data and medical guidelines. Transplantation. 2005; 79: S53-S66.

17. European Renal Best Practice Transplantation Guideline Development Group. ERBP guideline on the management and evaluation of the kidney donor and recipient. Nephrol Dial Transplant. 2013; 28 (Suppl. 2): ii1-ii71.

18. OPTN/UNOS. Organ Procurement \&amp; Transplantation Network Policy 14: living donation. OPTN. 2016. Disponible en: https://optn.transplant.hrsa.gov/media/1200/optn_policies. pdf

19. Huang N, Foster MC, Lentine KL, Garg AX, Poggio ED, Kasiske $\mathrm{BL}$ et al. Estimated GFR for Living Kidney Donor Evaluation. Am J Transplant. 2016; 16 (1): 171-180.

20. Gaillard F, Flamant M, Lemoine S, Baron S, Timsit MO, Eladari $D$ et al. Estimated or measured GFR in living kidney donors work-up? Am J Transplant. 2016; 16 (10): 3024-3032.

21. Garg AX, Muirhead N, Knoll G, Yang RC, Prasad GV, ThiessenPhilbrook $\mathrm{H}$ et al. Proteinuria and reduced kidney function in living kidney donors: A systematic review, meta-analysis, and meta-regression. Kidney Int. 2006; 70 (10): 1801-1810.

22. Kasiske BL, Anderson-Haag T, Israni AK, Kalil RS, Kimmel PL, Kraus ES et al. A prospective controlled study of living kidney donors: three-year follow-up. Am J Kidney Dis. 2015; 66 (1): 114-124.

23. Fehrman-Ekholm I, Nordén G, Lennerling A, Rizell M, Mjörnstedt $L$, Wramner $L$ et al. Incidence of end-stage renal disease among live kidney donors. Transplantation. 2006; 82 (12): 1646-1648.

24. Ibrahim HN, Foley R, Tan L, Rogers T, Bailey RF, Guo H et al. Long-term consequences of kidney donation. N Engl J Med. 2009; 360 (5): 459-469.

25. Cherikh WS, Young CJ, Kramer BF, Taranto SE, Randall $\mathrm{HB}$, Fan PY. Ethnic and gender related differences in the risk of end-stage renal disease after living kidney donation. Am J Transplant. 2011; 11 (8): 1650-1655.

26. Mjøen G, Hallan S, Hartmann A, Foss A, Midtvedt K, Øyen O et al. Long-term risks for kidney donors. Kidney Int. 2014; 86 (1): 162-167.

27. Muzaale AD, Massie AB, Wang MC, Montgomery RA, McBride $M A$, Wainright $J$ et al. Risk of end-stage renal disease following live kidney donation. JAMA. 2014; 311 (6): 579-586.

28. Rizvi SA, Zafar MN, Jawad F, Aziz T, Hussain Z, Hashmi A et al. Long-term Safety of Living Kidney Donation in an Emerging Economy. Transplantation. 2016; 100 (6): 1284-1293.

29. Fogazzi GB, Garigali G. Urinalysis. In: Feehally J, Floege J, Tonelli M, Johnson RJ. Comprehensive clinical nephrology. 6th ed. Elsevier; 2019. pp. 39-52.

30. Inker LA. Albuminuria: time to focus on accuracy. Am J Kidney Dis. 2014; 63 (3): 378-381.

31. Gansevoort RT, Verhave JC, Hillege HL, Burgerhof JG, Bakker SJ, de Zeeuw D et al. The validity of screening based on spot morning urine samples to detect subjects with microalbuminuria in the general population. Kidney Int Suppl. 2005; (94): S28-S35.

32. Grams ME, Sang Y, Levey AS et al. Kidney-failure risk projection for the living kidney-donor candidate. N Engl JMed. 2016; 374: 411-421.

33. Mandelbrot DA, Pavlakis M, Danovitch GM et al. The medical evaluation of living kidney donors: a survey of US transplant centers. Am J Transplant. 2007; 7: 2333-2343.

34. Davis R, Jones JS, Barocas DA et al. American Urological Association (AUA) guideline ADULTS: AUA guideline American Urological Association asymptomatic microhematuria. J Urol. 2012; 188 (6 Suppl): 2473-2481.

35. Kassouf W, Aprikian A, Black $P$ et al. Recommendations for the improvement of bladder cancer quality of care in Canada: $A$ consensus document reviewed and endorsed by Bladder Cancer Canada (BCC), Canadian Urologic Oncology Group (CUOG), and Canadian Urological Association (CUA), December 2015. Can Urol Assoc J. 2016; 10: E46-E80.

36. Cohen RA, Brown RS. Clinical practice. Microscopic hematuria. N Engl J Med. 2003; 348: 2330-2338.

37. Shen $P, H e ~ L$, Jiang $Y$, Wang $C$, Chen M. Useful indicators for performing renal biopsy in adult patients with isolated microscopic haematuria. Int J Clin Pract. 2007; 61 (5): 789-794.

38. Kelly JD, Fawcett DP, Goldberg LC. Assessment and management of non-visible haematuria in primary care. BMJ. 2009; 338: a3021.

39. Hole B, Whittlestone $\mathrm{T}$, Tomson $\mathrm{C}$. Investigating asymptomatic invisible haematuria. BMJ. 2014; 349: g6768.

40. D'Amico G. Natural history of idiopathic IgA nephropathy and factors predictive of disease outcome. Semin Nephrol. 2004; 24 (3): 179-196.

41. Savige J, Rana K, Tonna $S$ et al. Thin basement membrane nephropathy. Kidney Int. 2003; 64: 1169-1178.

42. Scales CD Jr, Smith AC, Hanley JM, Saigal CS; Urologic diseases in America project. Prevalence of kidney stones in the United States. Eur Urol. 2012; 62: 160-165.

43. Boyce CJ, Pickhardt PJ, Lawrence EM, Kim DH, Bruce RJ. Prevalence of urolithiasis in asymptomatic adults: objective 
determination using low dose non contrast computerized tomography. J Urol. 2010; 183 (3): 1017-1021.

44. Lorenz EC, Lieske JC, Vrtiska TJ et al. Clinical characteristics of potential kidney donors with asymptomatic kidney stones. Nephrol Dial Transplant. 2011; 26: 2695-2700.

45. Olsburgh J, Thomas K, Wong $\mathrm{K}$ et al. Incidental renal stones in potential live kidney donors: prevalence, assessment and donation, including role of ex vivo ureteroscopy. BJU Int. 2013; 111 (5): 784-792.

46. O'Neill DC, Davis NF, Murray TÉ, Lee MJ, Little D, Morrin MM. Prevalence of incidental findings on multidetector computed tomography in potential nephrectomy donors: a prospective observational study. Exp Clin Transplant. 2019; 17 (2): 177-182.

47. Kim IK, Tan JC, Lapasia J, Elihu A, Busque S, Melcher ML. Incidental kidney stones: a single center experience with kidney donor selection. Clin Transplant. 2012; 26 (4): 558-563.

48. Evan AP. Physiopathology and etiology of stone formation in the kidney and the urinary tract. Pediatr Nephrol. 2010; 25: 831-841.

49. Jungers $P$, Joly D, Barbey F, Choukroun G, Daudon M. ESRD caused by nephrolithiasis: prevalence, mechanisms, and prevention. Am J Kidney Dis. 2004; 44: 799-805.

50. Alexander RT, Hemmelgarn BR, Wiebe N, Bello A, Morgan C, Samuel $S$ et al. Kidney stones and kidney function loss: a cohort study. BMJ. 2012; 345: e5287.
51. El-Zoghby ZM, Lieske JC, Foley RN, Bergstralh EJ, Li X, Melton LJ 3rd et al. Urolithiasis and the risk of ESRD. Clin J Am Soc Nephrol. 2012; 7 (9): 1409-1415.

52. Gambaro G, Croppi E, Bushinsky D, Jaeger P, Cupisti A, Ticinesi $A$ et al. The risk of chronic kidney disease associated with urolithiasis and its urological treatments: a review. J Urol. 2017; 198 (2): 268-273.

53. Pearle MS, Goldfarb DS, Assimos DG et al. Medical management of kidney stones: AUA guideline. J Urol. 2014; 192: 316-324.

54. Serur D, Charlton M, Juluru K et al. Long term follow up of kidney donors with asymptomatic renal stones. Nephrology. 2017; 22: 649-651.

55. Sarier M, Duman I, Callioglu M, Soylu A, Tekin S, Turan E et al. Outcomes of conservative management of asymptomatic live donor kidney stones. Urology. 2018; 118: 43-46.

56. Sarier M, Duman I, Yuksel Y, Tekin S, Ozer M, Yucetin L et al. Ex vivo stone surgery in donor kidneys at renal transplantation. Int J Urol. 2018; 25 (10): 844-847.

\section{Correspondencia:}

Dra. Roxana Villca-Gonzáles

E-mail: roxanavillca@yahoo.com 